\title{
Dbx1b defines the dorsal habenular progenitor domain in the zebrafish epithalamus
}

\author{
Benjamin J Dean², Begum Erdogan', Joshua T Gamse',2 and Shu-Yu Wu*
}

\begin{abstract}
Background: The conserved habenular nuclei function as a relay system connecting the forebrain with the brain stem. They play crucial roles in various cognitive behaviors by modulating cholinergic, dopaminergic and serotonergic activities. Despite the renewed interest in this conserved forebrain region because of its importance in regulating aversion and reward behaviors, the formation of the habenular nuclei during embryogenesis is poorly understood due to their small size and deep location in the brain, as well as the lack of known markers for habenular progenitors. In zebrafish, the bilateral habenular nuclei are subdivided into dorsal and ventral compartments, are particularly large and found on the dorsal surface of the brain, which facilitates the study of their development.
\end{abstract}

Results: Here we examine the expression of a homeodomain transcription factor, $d b \times 1 b$, and its potential to serve as an early molecular marker of dorsal habenular progenitors. Detailed spatiotemporal expression profiles demonstrate that the expression domain of $d b \times 1 b$ correlates with the presumptive habenular region, and $d b \times 1 b$-expressing cells are proliferative along the ventricle. A lineage-tracing experiment using the Cre-lox system confirms that all or almost all dorsal habenular neurons are derived from $d b \times 1 b$-expressing cells. In addition, mutant analysis and pharmacological treatments demonstrate that both initiation and maintenance of $d b \times 1 b$ expression requires precise regulation by fibroblast growth factor (FGF) signaling.

Conclusions: We provide clear evidence in support of $d b \times 1 b$ marking the progenitor populations that give rise to the dorsal habenulae. In addition, the expression of $d b \times 1 b$ in the dorsal diencephalon is tightly controlled by FGF signaling.

\section{Background}

The habenular nuclei (habenulae) develop in the dorsal diencephalon of vertebrates. These bilaterally paired nuclei receive inputs from the limbic system and basal ganglia and send outputs to dopaminergic and serotonergic centers. Despite their small size, these nuclei play crucial roles in regulating aversion and reward behaviors [1]. Moreover, the fact that the habenulae are a nexus for monoamine circuits highlights the importance of this brain region for studies of neuromodulation and multicircuit integration.

The habenular nuclei can be divided into two functionally distinct subnuclei alternately referred to as medial and lateral (mammals) or dorsal and ventral (zebrafish) subnuclei.

\footnotetext{
* Correspondence: simon.wu@vanderbilt.edu

1 Department of Biological Sciences, Vanderbilt University, Box 351634 Station B, Nashville, TN 37235-1634, USA

Full list of author information is available at the end of the article
}

Recent study of the habenulae has largely focused on the ventral habenulae, but interest in the dorsal habenulae is increasing [2]. Alterations in dorsal habenular structure and function during development have been linked to dopamine receptor expression as well as impulsivity, attention, aversion and spatial memory endophenotypes in rodents $[3,4]$. In zebrafish, the dorsal habenulae regulate fear and aversion [5,6]. Together these studies implicate the dorsal habenulae as a possible mediator of attention deficit hyperactivity disorder (ADHD), depression and anxiety [2]. Interestingly, the dorsal habenulae are also sites of intense acetylcholine receptor and transporter expression and control nicotine intake [7-9]. This raises the possibility of targeting the habenulae as a therapeutic intervention to nicotine addiction. Beyond translational research, the zebrafish habenulae also serve as an excellent model to study the basic mechanisms underlying the development of left-right brain asymmetry. Unlike the 
mammalian habenulae, teleost dorsal habenulae are robustly asymmetric in anatomy, gene expression and functional connectivity [10].

Interest in how the dorsal habenulae integrate into cholinergic and monoaminergic circuitry has put pressure on researchers to understand dorsal habenular development. Indeed, Beretta et al. [11] have shown that dorsal and ventral habenular neurons arise from distinct progenitor populations. While progress has been made in describing habenular neurogenesis, differentiation and elaboration of processes, there are no known markers for habenular progenitors [12-16]. Therefore, finding marker genes that label dorsal habenular progenitors will be fundamental to studying how the diverse set of dorsal habenular neurons are generated and integrated into neural circuits underpinning aversive behavior as well as pathological addictive and depressive behaviors.

The $d b x$ homeodomain transcription factors play a central role in regulating progenitor status in several regions in the developing central nervous system, including the spinal cord $[17,18]$. However, the upstream regulatory pathways that regulate $d b x$ gene-family expression are not known. Here we report that in zebrafish, $d b \times 1 b$ is expressed in the dorsal diencephalon where it marks dorsal habenular progenitors, and further, that dorsal diencephalic expression of $d b \times 1 b$ is controlled by fibroblast growth factor (FGF) signaling.

\section{Results and discussion}

\section{$D b \times 1 b$ is expressed in the presumptive habenulae}

During our ongoing efforts to characterize transcription factors (TFs) that are expressed in the dorsal diencephalic region between 24 and 48 hours post-fertilization (hpf), we focused on a family of homeodomain-containing TFs encoded by the $d b x$ genes because of their known roles in neural progenitors. There are three $d b x$ genes in the zebrafish genome, and we carefully examined the expression pattern of the two $d b x 1$ paralogs, $d b x 1 a$ and $d b x 1 b$. We excluded $d b x 2$ from our study because its expression has been detailed previously [19]. At $28 \mathrm{hpf}, d b \times 1 a$ and $d b x 1 b$ showed similar yet distinct expression patterns (Additional file 1). As shown previously [20], dbxla was expressed in sharply restricted domains in the diencephalon with prominent expression in the prethalamus and thalamus (Additional file 1: panel A). Expression of $d b \times 1 a$ and $d b \times 1 b$ was similar in the prethalamic region, but in the thalamic region $d b x 1 b$ was expressed at a much lower level than $d b x 1 a$ (Additional file 1; panel C). A more striking difference between the patterns of these two paralogs was the expression of $d b \times 1 b$ in the dorsal diencephalon, where $d b x 1 a$ was completely absent (compare arrowheads in Additional file 1). Expression of $d b \times 1 b$ was excluded from the ot $x 5$-positive pineal complex, the other major structure of the dorsal diencephalon
(Additional file 1, panel $C$ ). These data suggested that $d b x 1 b$ could be an early molecular marker for the presumptive habenulae.

Next, we closely examined the expression of $d b x 1 b$ at different developmental stages (Figure 1). At $22 \mathrm{hpf}$, expression of $d b \times 1 b$ was not yet present in the presumptive habenular region, although the prethalamic and midbrain regions showed strong expression (Figure 1A,B). By $24 \mathrm{hpf}$, habenular $d b x 1 b$ expression appeared (Figure $1 C, \mathrm{D})$ and was maintained through at least $96 \mathrm{hpf}$ (Figure 1E-J and data not shown). Moreover, $d b \times 1 b$ expression remained highest adjacent to the third ventricle of the brain, and was absent from regions distal to the ventricle by 48 hpf (Figure $1 \mathrm{H}$ and $1 \mathrm{~J}$; dorsal views). Because neuronal progenitors are often found in regions adjacent to the ventricle in the developing brain, these data led us to speculate that the $d b x 1 b$ expression pattern includes the progenitors of habenular neurons.

\section{Dbx1b labels dorsal habenular progenitors}

To support the hypothesis that $d b \times 1 b$-expressing cells represent habenular progenitors, we examined if these cells are proliferative. Indeed, as shown by phospho-histone $\mathrm{H} 3$ staining at $32 \mathrm{hpf}$, the $d b x 1 b$-positive cells close to the ventricular surface were proliferative, which was consistent with progenitor cell identity (Figure 2A-D). To see if $d b x 1 b$ expression is restricted to progenitors we compared the expression of previously described precursor and neuronal habenular markers. $C x c r 4 b$ has been proposed as a marker of progenitors as well as post-mitotic habenular precursors [12]. At $36 \mathrm{hpf}$, a subset of $d b x 1 b$-expressing cells co-expressed $c x c r 4 b$. Specifically, $c x c r 4 b$ expression was restricted to the dorsal half of the $d b x 1 b$ expression domain leaving a ventral region of $d b x 1 b$-only expression along the ventricle (Figure 2E-F). At $48 \mathrm{hpf}$, Elavl3 (also known as $\mathrm{HuC}$ ) marks post-mitotic neurons. Expression of Elavl3 and $d b x 1 b$ was more distinct than $c x c r 4 b$ and $d b x 1 b$, with Elavl3 expressed dorsally and laterally while $d b \times 1 b$ was present medially and ventrally along the ventricle (Figure 2G-H). Kctd12.1 and Kctd12.2 mark different populations of differentiated habenular neurons. At 72 hpf, neither showed any overlap with $d b x 1 b$ expression (Figure 2I-L). Similar results were observed with another habenular differentiation marker, pou $4 f 1$ (or brn $3 a$ ) (data not shown). Together these results indicated that $d b \times 1 b$ expression is maintained in a proliferative population of habenular progenitor cells that reside along the ventricle. $D b x 1 b$ expression was absent in fully differentiated neurons, suggesting that $d b x 1 b$ is downregulated in mature habenular neurons. Thus, we conclude that $d b x 1 b$ can serve as an early marker to identify habenular progenitors.

To substantiate our conclusion that $d b x 1 b$ is expressed in habenular progenitors, we performed a lineage-tracing 

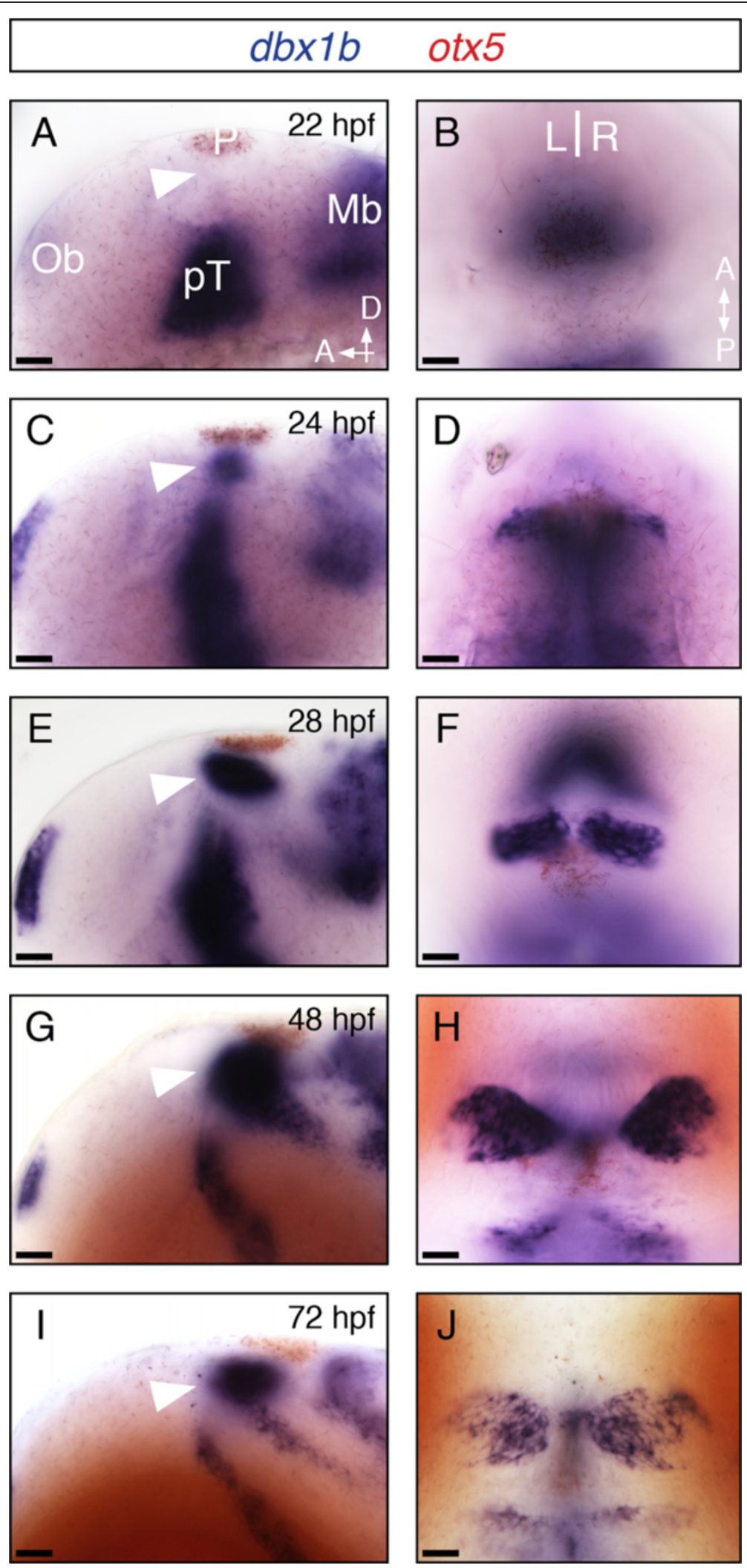

Figure $1 D b \times 1 b$ is expressed throughout habenular development. (A-J) Lateral and dorsal views of $d b \times 1 b$ expression (blue) during early brain development. Dorsal diencephalic expression of $d b \times 16$ appeared shortly after 22 hpf and continued through 72 hpf (arrowhead). otx5 (red) marks the pineal complex (P). Ob, olfactory bulb; PT, prethalamus; Mb, midbrain. Scale bars are $10 \mu \mathrm{m}$. 


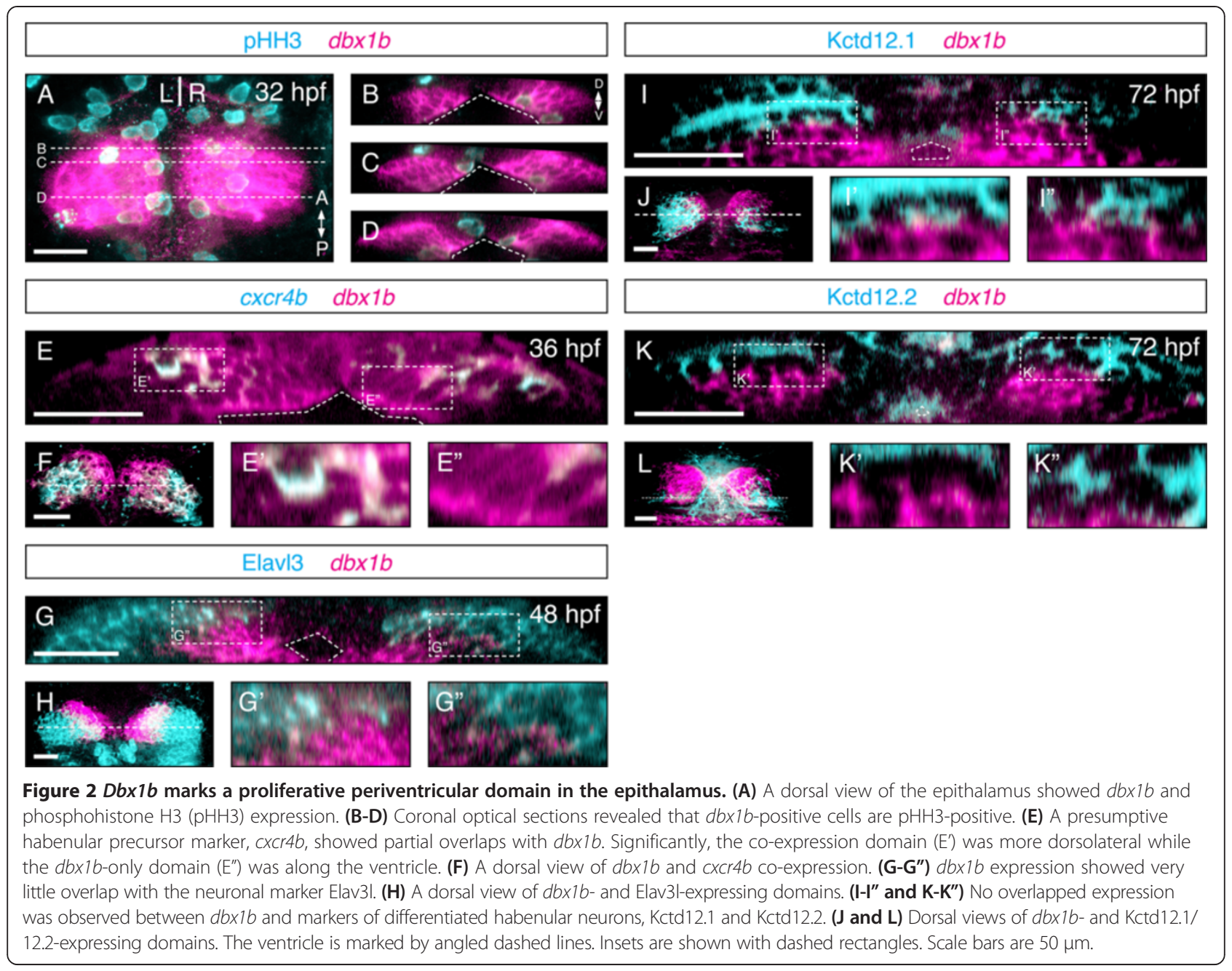

experiment using the Cre-lox recombination system. By crossing the TgBAC[dbx1b:Cre-mCherry] [21] transgenic line with a reporter line, $T g[-10 a c t b 2: L O X P$-mCherryLOXP-nlsEGFP] [22], almost all Elavl3-expressing habenular neurons were co-labeled with GFP (Figure 3A-C). Indeed, the only domain expressing GFP but not Elavl3 was found along the ventricle, in an area that coincides with $d b \times 1 b$ transcription (Figure 3D-G). To determine if $d b \times 1 b$ positive cells give rise to both dorsal and ventral habenular neurons, we compared the lineage-labeled domain with cadps2, a dorsal habenular marker [23]. Almost all lineagelabeled cells were cadps2-positive (Figure $3 \mathrm{H}-\mathrm{J}$ ). The only $d b \times 1 b$-derived cells (GFP-positive) outside the cadps 2 domain were medially located and along the ventricle; none were found laterally in the region of the ventral habenulae. Moreover, we also compared the lineage-labeled domain with the expression domain of aoc1, a known ventral habenular marker [24], and found that these two domains abutted but clearly did not overlap (Figure $3 \mathrm{~K}-\mathrm{M}$ ). In conclusion, these results strongly suggested that most if not all post-mitotic dorsal habenular neurons are derived from progenitor cells that express $d b x 1 b$ prior to their differentiation, confirming that $d b x 1 b$ is a marker of early dorsal habenular progenitors.

\section{FGF signaling is required for proper development of the} dorsal habenulae

FGF signaling has been shown to play critical roles during the development of the zebrafish dorsal diencephalon, particularly in pineal complex specification and parapineal migration $[25,26]$. It has been suggested that the development of both the left and right habenulae requires FGF signaling, as shown by the reduced expression of habenular differentiation markers (Kctd12.1, pou4f1) in FGF mutants [26]. However, how the loss of FGF signaling impacts habenular development remains unclear. We found that in $f g f 8 a$ mutants, in which brain patterning appeared to be normal (Additional file 2), the expression of $d b \times 1 b$ was completely lost at $24 \mathrm{hpf}$ (Figure 4A-D) and this loss of expression persisted at later stages. The latter observation suggested that the loss of FGFdependent $d b \times 1 b$ is not a result of developmental delay. 


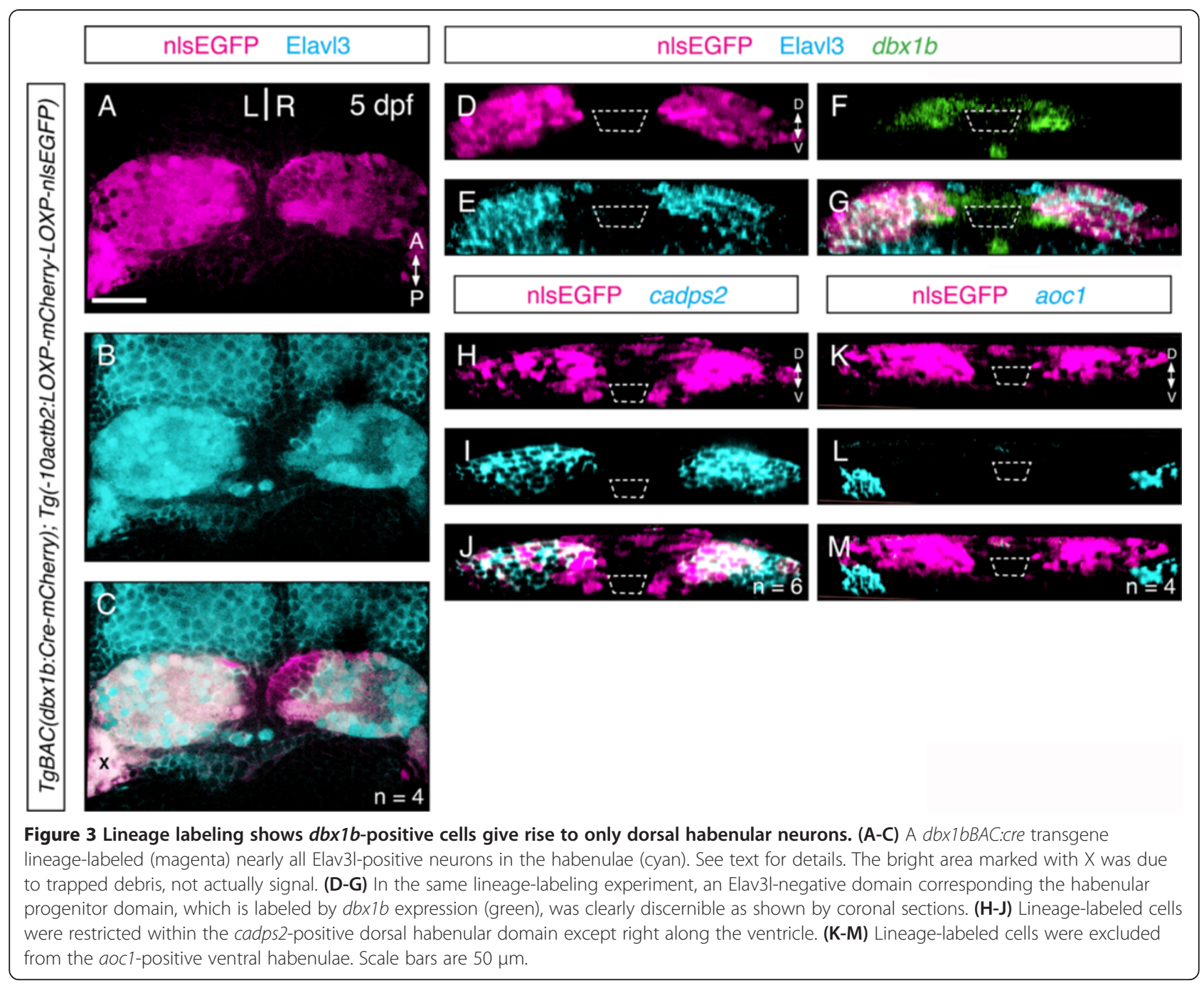

Therefore, FGF signaling is absolutely required for the initiation of $d b x 1 b$ expression.

To explore the regulatory relationship between FGF signaling and $d b \times 1 b$ in more detail, we investigated if FGF signaling is continuously required to maintain $d b \times 1 b$ positive habenular progenitors. We took advantage of the FGF receptor antagonist, SU5402, to block FGF signaling in a temporally controlled manner. Since $d b x 1 b$ expression was first detectable at $24 \mathrm{hpf}$, the embryos were treated with SU5402 for 8 hours, from either 28 to $36 \mathrm{hpf}$ or 48 to $56 \mathrm{hpf}$. The expression of $d b x 1 b$ was analyzed at the end of the treatments as well as after 12 hours of recovery post-treatment. As shown in Figure 5, both treatment regimes abrogated $d b x 1 b$ expression by the end of the treatment window, yet $d b x 1 b$ expression began to return after 12 hours of recovery (Figure 5B,D and 4F,H). This result suggests that FGF signaling is required for not only the initiation, but also the maintenance of $d b \times 1 b$ expression. Moreover, at least some neuronal progenitors in the dorsal diencephalon remain FGF-responsive and capable of reactivating $d b x 1 b$ expression upon exposure to FGF signal, even when they were previously deprived of FGF signaling.

\section{Conclusions}

This report describes the expression of $d b x 1 b$, which we believe is the first reported marker of the neuronal progenitors that give rise to the dorsal habenulae. In addition, we found that FGF signaling controls the expression of $d b x 1 b$ in the dorsal diencephalon. Together with other existing genetic tools, including various $d b \times 1 b$ BAC transgenic lines, our discovery of $d b \times 1 b$ as a habenular progenitor marker will not only allow for more detailed and nuanced investigation of dorsal habenular development, but also provide an exciting way forward to study proliferation, specification and circuit formation of the diverse neuronal populations in the habenular nuclei, and how these processes influence developmental and adult habenulae-mediated behaviors. 


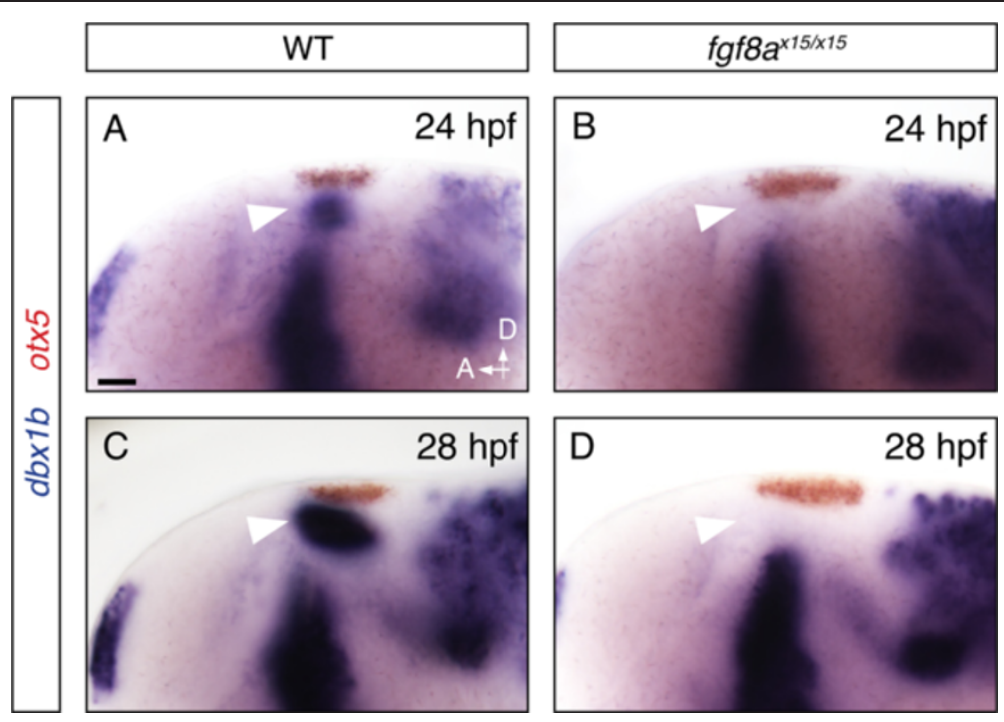

Figure 4 Fgf8a mutants fail to express $d b \times 1 b$ in the epithalamus. (A-D) In situ hybridization for $d b \times 1 b$ (blue) in wildtype and fgf8a mutant embryos. otx5 marks the pineal complex. Scale bars are $10 \mu \mathrm{m}$.

\section{Methods}

\section{Zebrafish maintenance and strains}

Zebrafish were raised at $28.5^{\circ} \mathrm{C}$ on a $14 / 10$ hour light/ dark cycle and staged according to hours post-fertilization. The following fish lines were used: the wildtype strain $\mathrm{AB}^{*}$ [27], TgBAC[dbx1b:Cre-mCherry] ${ }^{\text {nns } 13 a}$ [21] and $T g$ [-10actb2:LOXP-mCherry-LOXP-nlsEGFP $]^{\text {pd31 }}[22]$. All experiments were approved by the Vanderbilt University's Institutional Animal Care and Use Committee (IACUC) and Office of Animal Welfare, and performed according to national regulatory standards.

\section{Whole-mount in situ hybridization}

Whole-mount RNA in situ hybridization was performed as described previously [23], with one change: $5 \%$ dextran sulfate was added to the hybridization buffer to enhance hybridization specificity [28]. Hybridized probes were detected using alkaline phosphatase conjugated antibodies (Roche, Indianapolis, IN, USA) and visualized by 4-nitro blue tetrazolium (NBT; Roche) and 5-bromo-4-chloro3-indolyl-phosphate (BCIP; Roche) staining for single colorometric labeling, or by NBT/BCIP followed by iodonitrotetrazolium (INT) and BCIP staining for double colorometric labeling. $d b x 1 a$ probe [17] was produced from pCRII- $d b x 1 a$ plasmid linearized by EcoRV and transcribed by SP6 RNA polymerase. $d b x 1 b$ probe [17] using pCRII$d b x 1 b$, BamHI and T7 RNA polymerase, and otx5 [29] using pBS-otx5, Not1 and T7 RNA polymerase.

\section{Whole-mount fluorescent in situ hybridization and immunohistochemistry}

Whole-mount fluorescent in situ hybridization and immunohistochemical co-labeling was performed as described previously [13], with the following additional reagents: in addition to Fast Red substrate (F4648; Sigma-Aldrich, St Louis, MO, USA), some experiments used $3 \times 5$ minute washes in Fast Blue Buffer [28] and were developed in Fast Blue Substrate $(0.25 \mathrm{mg} / \mathrm{mL}$ Fast Blue Substrate and 0.25 $\mathrm{mg} / \mathrm{mL}$ nAMP in Fast Blue Buffer) diluted in Fast Blue Buffer. In addition to the anti-digoxigenin (DIG) antibody, the primary antibodies used were rabbit anti-pHH3 (1:500, EMD Millipore, Billerica, MA, USA), mouse anti-HuC (1:400, Life Technologies, Carlsbad, CA, USA), rabbit anti-GFP (1:500, Torrey Pines Biolab, Secaucus, NJ, USA), rabbit anti-Kctd12.1 and rabbit anti-Kctd12.2 (1:300, see [30]). Primary antibody was detected using goat-antirabbit or goat-anti-mouse antibodies conjugated to Alexa 488, Alexa 568 or Alexa 633 fluorophores (1:300, Molecular Probes, Eugene, OR, USA).

Double fluorescent in situ hybridization was performed with the following modifications to the above colorometric in situ protocol: after hybridization of DIG and fluorescein-labeled probes, anti-DIG antibody was applied (1:5,000, Roche) overnight at $4^{\circ} \mathrm{C}$. The following day, embryos were washed $4 \times 20$ minutes in PBS with Triton (PBSTr) and $3 \times 5$ minutes in Fast Blue Buffer and developed in Fast Blue Substrate diluted in Fast Blue Buffer. After color development, embryos were washed $2 \times 10$ minutes in PBSTr. The alkaline phosphatase was acid inactivated by a 10-minute wash in 0.1 $\mathrm{M}$ glycine $\mathrm{HCl}, \mathrm{pH} 2.0$. After $2 \times 10$ minute PBSTr washes, embryos were incubated in anti-fluorescein antibody $\left(1: 1,000\right.$, Roche) overnight at $4^{\circ} \mathrm{C}$. The following day, color was developed in Fast Red substrate as in Doll et al. [13]. cxcr4b [31] probe was generated with EcoRV and SP6 RNA polymerase. 


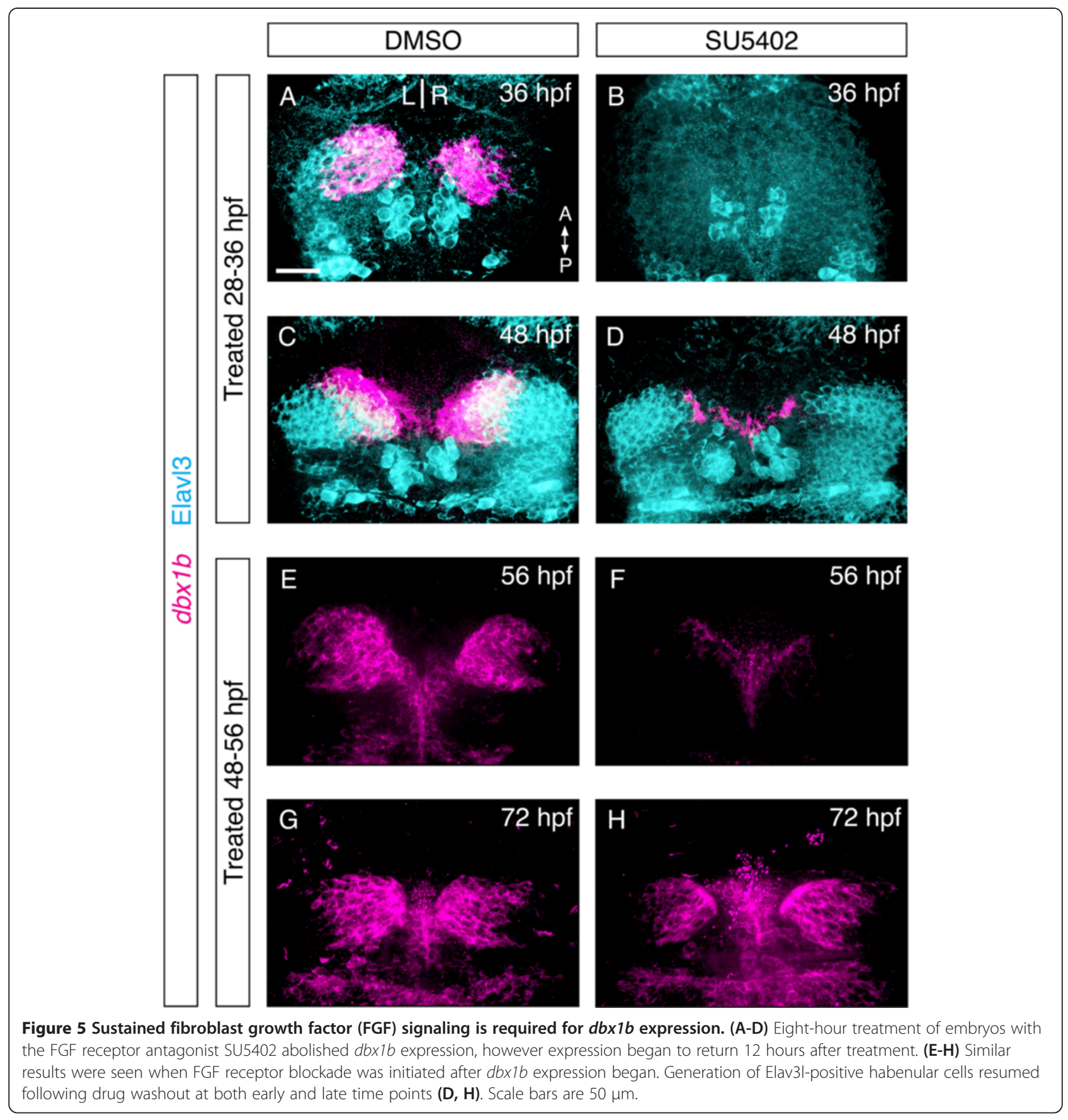

\section{Inhibitor treatments}

For whole-mount in situ hybridizations and antibody labeling, embryos were incubated in their chorions in $12 \mu \mathrm{M}$ (for complete receptor inhibition; [27]) of SU5402 (Tocris Bioscience, Bristol, UK) dissolved in 0.3\% dimethyl sulfoxide (DMSO) in egg water supplemented with $0.003 \%$ N-phenylthiourea (PTU; Sigma-Aldrich) to prevent melanin formation. Control embryos were treated with $0.3 \%$ DMSO in parallel with their SU5402-treated siblings. Embryos were either fixed immediately following treatment or SU5402/DMSO was washed off with $5 \times 5$ minute egg water before being returned to egg water with PTU to develop to the desired stage for fixation.

\section{Imaging}

All samples were cleared in a glycerol series (50\%, 100\%). Colorometric in situ images were captured on a Leica DM6000 B compound microscope (Leica Microsystems, Buffalo Grove, IL, USA) under a 20x air objective in bright field conditions. Fluorescent images were captured on a 
PerkinElmer spinning disk confocal microscope (PerkinElmer, Waltham, MA, USA) or a Zeiss LSM 510 Meta confocal microscope (Carl Zeiss Microscopy, Oberkochen, Germany) with a $40 \times$ oil-immersion objective and analyzed with Volocity software (Improvision/PerkinElmer, Waltham, MA, USA).

\section{Additional files}

Additional file 1: $D b \times 1 b$ is expressed in the dorsal diencephalon. Lateral and dorsal views of a $28 \mathrm{hpf}$ wildtype embryo. (A and B) In situ hybridization for $d b \times 1 a$ (blue) revealed several expression domains, including the olfactory bulb (Ob), prethalamus (pT), thalamus (Th), and midbrain (Mb) throughout the brain, but no expression in the dorsal diencephalon (arrow heads). (C and D) $d b \times 1 b$ transcript (blue) was expressed in a similar pattern but with greatly reduced expression in thalamus and robust expression in the dorsal diencephalon and olfactory bulb. ot $\times 5$ (red) marks the pineal complex (P), a component of the dorsal diencephalon. Scale bars are $10 \mu \mathrm{m}$.

Additional file 2: Fgf8a mutants show normal overall brain patterning. (A-B) In fgfia mutants there were no major anterior-posterior patterning defects observed. eomes, shh and wht 1 mark the telencephalon (Tel), zona limitans intrathalamica (ZLI) and midbrain (Mb) respectively. otx5 marks the pineal complex. (C-D) Dorsal-ventral patterning was also unaffected in fgf8a mutants. wnt3a (blue) marks the ZLI and midbrain and shh (red) marks the ZLI. Scale bars are $10 \mu \mathrm{m}$.

\section{Abbreviations}

actb2: actin, beta 2; ADHD: attention deficit hyperactivity disorder; aoc1: amine oxidase, copper containing 1; BAC: bacterial artificial chromosome BCIP: 5-bromo-4-chloro-3-indolyl-phosphate; cadps2: $\mathrm{Ca}^{2+}$-dependent secretion activator 2; cxcr4b: chemokine (C-X-C motif), receptor $4 \mathrm{~b}$; dbx1b: developing brain homeobox 1b; DIG: digoxigenin; dpf: days post-fertilization; DMSO: dimethyl sulfoxide; Elavl3: ELAV like neuron-specific RNA binding protein 3; eomesa: eomesodermin homolog a; FGF: fibroblast growth factor; GFP: green fluorescent protein; hpf: hours post-fertilization; HuC: Hu-antigen C; IACUC: Institutional Animal Care and Use Committee; Kctd12: potassium channel tetramerization domain containing 12; NBT: 4-nitro blue tetrazolium; INT: iodonitrotetrazolium; PBS: phosphatebuffered saline; otx5: orthodenticle homolog 5; pou4f1: POU domain, class 4, transcription factor 1 (also called Brain-specific homeobox/POU domain protein 3a, brn3a); PTU: N-phenylthiourea; shh: sonic hedgehog; TFs: transcription factors; Tg: transgenic; wnt1: wingless-type MMTV integration site family, member 1; ZLI: zona limitans intrathalamica.

\section{Competing interests}

The authors declare that they have no competing interests.

\section{Authors' contributions}

$\mathrm{BD}$ conceived and designed the study, undertook data collection and analysis and wrote the manuscript. BE undertook data collection. JG conceived and designed the study, arranged financial support and wrote the manuscript. SW conceived and designed the study, undertook data collection and analysis, and wrote the manuscript. All authors read and approved the final manuscript.

\section{Acknowledgements}

We would like to thank Gamse lab members for helpful discussions. We acknowledge the SC Vanderbilt University Fish Facility Research Assistants for excellent fish care. Experiments were performed in part through the use of the VUMC Cell Imaging Shared Resource (supported by NIH grants CA68485, DK20593, DK58404, HD15052, DK59637 and EY08126). This work was supported by Public Health Service award T32 GM07347 from the National Institute of General Medical Studies for the Vanderbilt Medical-Scientist Training Program to BJD, NIH grants F32HD069148 to SW, and R01HD054534 to JTG.

\section{Author details}

'Department of Biological Sciences, Vanderbilt University, Box 351634 Station B, Nashville, TN 37235-1634, USA. ${ }^{2}$ Program in Neuroscience, Vanderbilt University, Nashville, TN 37232, USA.

Received: 3 June 2014 Accepted: 1 September 2014 Published: 12 September 2014

\section{References}

1. Velasquez KM, Molfese DL, Salas R: The role of the habenula in drug addiction. Front Hum Neurosci 2014, 8:174

2. Viswanath $H$, Carter AQ, Baldwin PR, Molfese DL, Salas R: The medial habenula: still neglected. Front Hum Neurosci 2013, 7:931.

3. Lee Y-A, Goto Y: Neurodevelopmental disruption of cortico-striatal function caused by degeneration of habenula neurons. PLOS One 2011, 6:e19450.

4. Kobayashi Y, Sano Y, Vannoni E, Goto H, Suzuki H, Oba A, Kawasaki H, Kanba S, Lipp H-P, Murphy NP, Wolfer DP, Itohara S: Genetic dissection of medial habenula-interpeduncular nucleus pathway function in mice. Front Behav Neurosci 2013, 7:17.

5. Agetsuma M, Aizawa H, Aoki T, Nakayama R, Takahoko M, Goto M, Sassa T, Amo R, Shiraki T, Kawakami K, Hosoya T, Higashijima S-I, Okamoto H: The habenula is crucial for experience-dependent modification of fear responses in zebrafish. Nat Neurosci 2010, 13:1354-1356.

6. Mathuru AS, Jesuthasan S: The medial habenula as a regulator of anxiety in adult zebrafish. Front Neural Circuits 2013, 7:99.

7. Hong E, Santhakumar K, Akitake CA, Ahn SJ, Thisse C, Thisse B, Wyart C, Mangin J-M, Halpern ME: Cholinergic left-right asymmetry in the habenulo-interpeduncular pathway. Proc Natl Acad Sci U S A 2013, 110:21171-21176.

8. Fowler CD, Lu Q, Johnson PM, Marks MJ, Kenny PJ: Habenular a5 nicotinic receptor subunit signalling controls nicotine intake. Nature 2011, 471:597-601.

9. Frahm S, Slimak MA, Ferrarese L, Santos-Torres J, Antolin-Fontes B, Auer S, Filkin S, Pons S, Fontaine J-F, Tsetlin V, Maskos U, Ibañez-Tallon I: Aversion to nicotine is regulated by the balanced activity of $\beta 4$ and a5 nicotinic receptor subunits in the medial habenula. Neuron 2011, 70:522-535

10. Bianco $\mathrm{H}$, Wilson SW: The habenular nuclei: a conserved asymmetric relay station in the vertebrate brain. Philos Trans $R$ Soc Lond B Biol Sci 2009, 364:1005-1020.

11. Beretta CA, Dross N, Bankhead P, Carl M: The ventral habenulae of zebrafish develop in prosomere 2 dependent on Tcf7l2 function. Neural Dev 2013, 8:19.

12. Roussigné $\mathrm{M}$, Bianco $\mathrm{H}$, Wilson SW, Blader P: Nodal signalling imposes left-right asymmetry upon neurogenesis in the habenular nuclei. Development 2009, 136:1549-1557.

13. Doll CA, Burkart JT, Hope KD, Halpern ME, Gamse JT: Subnuclear development of the zebrafish habenular nuclei requires ER translocon function. Dev Biol 2011, 360:44-57.

14. Aizawa H, Goto M, Sato T, Okamoto H: Temporally regulated asymmetric neurogenesis causes left-right difference in the zebrafish habenular structures. Dev Cell 2007, 12:87-98.

15. DeCarvalho TN, Subedi A, Rock J, Harfe BD, Thisse C: Neurotransmitter map of the asymmetric dorsal habenular nuclei of zebrafish. Genesis 2014, $52: 1-42$.

16. Taylor RW, Qi JY, Talaga AK, Ma TP, Pan L, Bartholomew CR, Klionsky DJ, Moens CB, Gamse JT: Asymmetric inhibition of Ulk2 causes left-right differences in habenular neuropil formation. J Neurosci 2011, 31:9869-9878.

17. Gribble SL, Nikolaus OB, Dorsky Rl: Regulation and function of Dbx genes in the zebrafish spinal cord. Dev Dyn 2007, 236:3472-3483.

18. Vue TY, Aaker J, Taniguchi A, Kazemzadeh C, Skidmore JM, Martin DM, Martin JF, Treier M, Nakagawa Y: Characterization of progenitor domains in the developing mouse thalamus. J Comp Neurol 2007, 505:73-91.

19. Seo $\mathrm{H}-\mathrm{C}$, Nilsen F, Fjose A: Three structurally and functionally conserved HIx genes in zebrafish. Biochim Biophys Acta 1999, 1489:323-335.

20. Lauter G, Söll I, Hauptmann G: Molecular characterization of prosomeric and intraprosomeric subdivisions of the embryonic zebrafish diencephalon. J Comp Neurol 2013, 521:1093-1118. 
21. Koyama M, Kinkhabwala A, Satou C, Higashijima S, Fetcho J: Mapping a sensory-motor network onto a structural and functional ground plan in the hindbrain. Proc Natl Acad Sci U S A 2011, 108:1-6.

22. Kikuchi K, Holdway JE, Werdich AA, Anderson RM, Fang Y, Egnaczyk GF, Evans T, Macrae CA, Stainier DYR, Poss KD: Primary contribution to zebrafish heart regeneration by gata4(+) cardiomyocytes. Nature 2010 464:601-605.

23. Gamse JT: The parapineal mediates left-right asymmetry in the zebrafish diencephalon. Development 2003, 130:1059-1068.

24. Amo R, Aizawa H, Takahoko M, Kobayashi M, Takahashi R, Aoki T, Okamoto H: Identification of the zebrafish ventral habenula as a homolog of the mammalian lateral habenula. J Neurosci 2010, 30:1566-1574.

25. Clanton JA, Hope KD, Gamse JT: Fgf signaling governs cell fate in the zebrafish pineal complex. Development 2013, 140:323-332.

26. Regan JC, Concha ML, Roussigne M, Russell C, Wilson SW: An Fgf8-dependent bistable cell migratory event establishes CNS asymmetry. Neuron 2009, 61:27-34.

27. Walker C: Haploid screens and gamma-ray mutagenesis. Methods Cell Biol 1999, 60:43-70

28. Lauter G, Söll I, Hauptmann G: Multicolor fluorescent in situ hybridization to define abutting and overlapping gene expression in the embryonic zebrafish brain. Neural Dev 2011, 6:10

29. Gamse JT, Shen Y-C, Thisse C, Thisse B, Raymond PA, Halpern ME, Liang JO: Otx5 regulates genes that show circadian expression in the zebrafish pineal complex. Nat Genet 2002, 30:117-121.

30. Gamse JT, Kuan Y-S, Macurak M, Brösamle C, Thisse B, Thisse C, Halpern ME: Directional asymmetry of the zebrafish epithalamus guides dorsoventral innervation of the midbrain target. Development 2005, 132:4869-4881.

31. Chong SW, Emelyanov A, Gong Z, Korzh V: Expression pattern of two zebrafish genes, cxcr4a and cxcr4b. Mech Dev 2001, 109:347-354.

doi:10.1186/1749-8104-9-20

Cite this article as: Dean et al: Dbx1b defines the dorsal habenular progenitor domain in the zebrafish epithalamus. Neural Development 2014 9:20.

\section{Submit your next manuscript to BioMed Central and take full advantage of:}

- Convenient online submission

- Thorough peer review

- No space constraints or color figure charges

- Immediate publication on acceptance

- Inclusion in PubMed, CAS, Scopus and Google Scholar

- Research which is freely available for redistribution 